\title{
Désaccords dans un Duo. Un mouvement de la vie psychique à travers L'enfant et les sortilèges
}

\section{Laurence Lacour}

\section{(2) OpenEdition}

\section{Journals}

Édition électronique

URL : http://journals.openedition.org/edl/1156

DOI : $10.4000 /$ edl. 1156

ISSN : 2296-5084

Éditeur

Université de Lausanne

\section{Édition imprimée}

Date de publication : 15 décembre 2016

Pagination : 111-130

ISBN : ISBN 978-2-940331-50-5

ISSN : 0014-2026

\section{Référence électronique}

Laurence Lacour, « Désaccords dans un Duo. Un mouvement de la vie psychique à travers L'enfant et les sortilèges », Études de lettres [En ligne], 4 | 2016, mis en ligne le 15 décembre 2019, consulté le 15 décembre 2020. URL : http://journals.openedition.org/edl/1156 ; DOI : https://doi.org/10.4000/edl. 1156

\section{(c) Études de lettres}




\section{DÉSACCORDS DANS UN DUO. UN MOUVEMENT DE LA VIE PSYCHIQUE À TRAVERS L'ENFANT ET LES SORTILËGES}

L'enfant et les sortilèges est une fantaisie lyrique en deux parties écrite par Colette et mise en musique par Maurice Ravel. Explorant avec poésie et audace le monde de l'enfance, cet opéra enchanteur peut se lire sous l'angle de la psychanalyse, comme une illustration subtile et pertinente des processus psychiques présents dès le début de la vie dans la relation mère-enfant. Du duo chahuté de l'Enfant en conflit avec sa mère au duo inventif formé par Colette et Ravel, l'enjeu est peut-être le même: celui de la création comme un moyen de dépasser la souffrance humaine.

\section{En préambule}

Le 21 mars 1925 à l'opéra de Monte-Carlo, eut lieu la première représentation de L'enfant et les sortilèges, une fantaisie lyrique née de la plume de Colette et mise en musique par Maurice Ravel. Imaginé à une époque encore marquée par un contexte social très conservateur et une première guerre mondiale dévastatrice, l'opéra de Ravel et de Colette est aussi et surtout une œuvre des "années folles", autrement dit un exemple magnifique de la créativité de l'époque ainsi que de sa curiosité à l'égard du psychisme humain et de son fonctionnement inconscient.

L'histoire de cet opéra raconte la situation d'un enfant tiraillé entre ses désirs et les demandes de sa mère qu'il n'arrive pas à satisfaire. Envahi par l'angoisse que créent ces tensions et débordé par sa frustration et sa colère à l'égard de sa mère, l'enfant sombre alors dans un univers fantastique, peuplé d'objets animés et d'animaux inquiétants qui le mettent face 
à sa destructivité et l'incitent à trouver en lui les moyens de s'apaiser. Poétique, onirique et inventif, l'opéra de Ravel et de Colette peut aussi se lire comme une description très fine des processus du développement de l'enfant. Il est d'ailleurs surprenant de voir à quel point le récit imaginé par Colette décrit avec précision ce que des psychanalystes à la même époque cherchaient à conceptualiser. Le début de $\mathrm{XX}^{\mathrm{e}}$ siècle, en effet, est marqué par les nouvelles connaissances dans le domaine de la psychologie, essentiellement à partir des travaux de Sigmund Freud, créateur de la psychanalyse, qui met en avant le concept d'inconscient comme élément fondamental de l'univers psychique humain. Cette approche offre alors des perspectives de compréhension qui donnent une place importante aux émotions, aux rêves, aux fantasmes, aux désirs et aux angoisses présents dans chaque individu. L'homme n'est plus gouverné par la raison, mais il est bien un être divisé entre son soi conscient et raisonnable et son monde inconscient qui obéit à une toute autre logique. Si les travaux du neurologue autrichien traitent des adultes et théorisent surtout la question du père et de son autorité dans le développement de l'individu, d'autres psychanalystes vont s'intéresser directement aux enfants et au lien primaire qui se tisse entre le bébé et sa mère.

Il en est ainsi du travail de Mélanie Klein, psychanalyste britannique d'origine autrichienne, pionnière dans le travail avec de jeunes enfants, qui développera des théories sur le fonctionnement psychique du bébé encore reconnues à l'heure actuelle. En 1929, elle s'intéresse d'ailleurs à une description de L'enfant et les sortilèges, lorsque celui-ci est donné à Vienne, et en tire une première analyse qui figure dans un chapitre de son livre Essais de psychanalyse paru bien des années plus tard, en $1947^{1}$. La proposition d'une lecture psychanalytique de l'œuvre de Colette et de Ravel a donc bien tout son sens, tant par le contexte historique dans lequel elle fut créée que par son contenu, qui traite à l'évidence des fantasmes d'un enfant en relation avec sa mère. Du rôle de la mère de "L'Enfant" sous l'emprise des «sortilèges" à la place de la mère dans la vie de Colette et même dans la vie de Ravel, il n'y a d'ailleurs qu'un petit pas à franchir. La lecture de leurs biographies respectives permet ainsi de mieux comprendre les liens que l'on peut faire entre l'œuvre et ses auteurs et de saisir les contextes dans lesquels cette création a pris place. Car ces deux artistes, que tout semblait séparer sur certains points et qui

I. M. Klein, Essais de psychanalyse 1921-1945, p. 254-259. 
ne se sont jamais rencontrés pour composer cet opéra, ont certainement pu trouver dans cette collaboration à distance des intérêts communs; leur amour pour la nature et les animaux, pour le merveilleux et l'audace, mais aussi un élan créatif autour d'une histoire porteuse d'un sens profond pour tous les deux. C'est en tout cas le sentiment que l'on peut avoir à l'analyse de cette œuvre...

\section{Les auteurs}

Sidonie Gabrielle Colette est la cadette d'une fratrie de quatre enfants, née en 1873 (-1954). Elle est adorée par sa mère qui la considérait comme "un joyau tout en or». Elle perd celle-ci en septembre 1912 et met au monde sa première et unique fille, Colette De Jouvenel dite «Bel-Gazou ", en juillet 1913, à l'âge de $40 \mathrm{ans}^{2}$. Colette mène une vie extraordinaire, sulfureuse pour les uns, libérée et moderne pour les autres, entre music-hall, journalisme, carrière d'écrivain et passions amoureuses. A côté de cet exubérant parcours de femme, sa maternité apparaît comme une expérience plus complexe voire compliquée, marquée dans les faits par de longues séparations d'avec sa fille. La première a lieu dans le contexte de la guerre, puisqu'elle décide de placer BelGazou, encore bébé, dans le château familial en Corrèze entre 1914 et 1918 et la placera par la suite en pension ${ }^{3}$. C'est donc en 1916, dans ce contexte de séparation mère-enfant et dans un temps probablement encore marqué par la perte de sa propre mère que Colette écrit le livret de L'enfant et les sortilèges sur demande du directeur de l'Opéra de Paris de l'époque, Jacques Rouché. Elle l'intitule d'ailleurs «Ballet pour ma petite fille» ${ }^{4}$. C'est ce même directeur qui propose à Colette de solliciter Maurice Ravel pour la partie musicale de son ouvre comme elle le rappelle elle-même dans ses souvenirs:

Vint le jour où M. Rouché (directeur de l'Opéra) me demanda un livret de féerie-ballet pour l'Opéra. Je ne m'explique pas encore comment je lui donnai, moi qui travaille avec lenteur et peine, L'enfant et les

2. M. Delcroix, «De la maison Sido à la maison de Claudine», p. 13-18.

3. C. et V. Pichois, "Album Colette».

4. R. Pourvoyeur, "Sortilèges de Colette et Ravel», p. 18-22. 
sortilèges en moins de huit jours... Il aima mon petit poème et suggéra des compositeurs dont j'accueillis le nom aussi poliment que je le pus. «Mais, dit Rouché après un silence, si je vous proposais Ravel?».Je sortis bruyamment de ma politesse et l'expression de mon espoir ne ménagea plus rien. "Il ne faut pas nous dissimuler, ajouta Rouché, que cela peut être long, en admettant que Ravel accepte...»5.

Maurice Ravel, né en 1875 (-1937), est le fils aîné d'un ingénieur suisse, issu d'une famille d'horlogers, et d'une mère originaire d'Espagne, tous deux amateurs de musique. Mis au piano à l'âge de 6 ans, il grandit donc avec la musique, entre au conservatoire à 14 ans et commence à composer vers l'âge de 18 ans. Soutenu et guidé par Gabriel Fauré, il développe ses talents de créateur et rencontre dans son évolution artistique bon nombre d'admirateurs, mais aussi des détracteurs. Souvent comparé à Debussy, parfois rangé "parmi les fabricants de boîtes à musique de luxe, destinées à l'amusement d'une élite de connaisseurs " ${ }^{6}$, Ravel réussit pourtant à allier sa technique sans faille à une créativité hors du commun. De ses origines, il garde la passion des petits automates dont il fait collection et des goûts musicaux sans doute influencés par les origines hispaniques de sa mère. Mais il va puiser également dans des sonorités orientales, exotiques et dans le jazz, ce qui fait de lui un compositeur classique, perfectionniste, mais aussi inventif et moderne:

Chaque œuvre du musicien de Shéhérazade est une expérience nouvelle, une prospection dans des gisements sonores inconnus et une réussite. [...] Le visage qu'il montre est toujours, au génie près qui est immuable, un visage inattendu?

En contraste avec l'audace de sa musique et la vie tumultueuse de Colette, Maurice Ravel est plutôt décrit comme un homme solitaire, discret, distingué et pudique qui se livre très peu. Il ne se mariera jamais et choisira de vivre seul entouré de nombreux chats qu'il adorait ${ }^{8}$. Exempté de service militaire en raison de sa stature fragile (petit et malingre), il décide tout de même de s'engager dans l'armée en 1916 et se retrouve

5. Colette et al., Maurice Ravel par quelques-uns de ses familiers, p. 120.

6. E. Vuillermoz, «Maurice Ravel», p. 112.

7. R. Brussel, «Ravel, classique français», p. 73.

8. D. Sanson «Maurice Ravel», p. 130. 
au front à Verdun. Il tombe alors malade et doit être opéré d'une péritonite. Il apprend également le décès de sa mère en janvier 1917, puis il est démobilisé en mars de la même année. C’est justement dans cette période, que Colette envoie le livret à Ravel. Un premier exemplaire se perd et Ravel reçoit finalement le texte en 1918. Mais affaibli, effondré par l'expérience du front et le décès de sa mère, il le laisse de côté pour se remettre à l'écriture de l'oeuvre en 1924, sous la pression d'un contrat avec l'Opéra de Monte-Carlo. Quelques mois lui suffisent alors pour écrire sa partition et L'enfant et les sortilèges est représenté pour la première fois au printemps $1925^{\circ}$.

\section{Une proposition d'analyse}

L'histoire que nous proposent Colette et Ravel se structure en deux parties et commence dans la maison de l'Enfant, pour se poursuivre et se terminer dans le jardin animé par ses arbres et ses animaux. Elle commence ainsi par une scène bien ordinaire, qui ne laisse rien soupçonner (ou presque) des évènements fantastiques qui vont suivre.

Un enfant, fille ou garçon (le genre ne sera jamais précisé dans le texte) de 6-7 ans rechigne à faire ses devoirs. Il se plaint et laisse libre cours à ses pensées qui ressemblent fort à des envies de faire des bêtises. Sa mère arrive alors pour vérifier son travail et le réprimande. Pour toute réponse l'enfant lui tire la langue. La mère se fâche et le punit: l'enfant sera privé d'un bon goûté et devra rester seul jusqu'au dîner. La mère disparaît. L'enfant laissé face à lui-même est pris d'une rage incontrôlable: il balaie la théière et la tasse, ouvre la cage d'un écureuil qu'il cherche à piquer avec sa plume, tire la queue du chat, tisonne le feu de la cheminée, déchire la tapisserie, arrache le balancier de l'horloge, arrache les pages de son livre et hurle sa fureur: «Hourrah! Plus de leçons! Plus de devoirs! Je suis libre, libre méchant et libre!». Essoufflé par sa frénésie, l'enfant s'affale sur un gros fauteuil, mais celui-ci se dérobe, s'anime et se met à parler. L'enfant se retrouve ainsi au sol, sidéré devant ce monde étrange qui s'offre à lui. Les objets qu'il a maltraités vont alors tour à tour se mettre à bouger et à parler pour le réprimander et le menacer. L'horloge, la tasse et la théière s'animent, mais aussi le feu, les personnages de la tapisserie

9. R. Pourvoyeur, «Sortilèges de Colette et Ravel», p. 22 
déchirée, la princesse du livre de conte de fée et même l'arithmétique, incarné par un petit vieillard «bossu, crochu, barbu et vêtu de chiffres". Il scande alors des bribes de problèmes mathématiques qui ont perdu toute logique et font tourner la tête à l'enfant. C'est ensuite au tour du chat d'entamer un duo miaulé inquiétant auprès de la chatte. L'enfant terrorisé observe cet étrange spectacle et connaît un bref moment de soulagement lorsqu'il retrouve le jardin. Mais celui-ci aussi s'exprime et rappelle à l'enfant toutes les blessures qu'il a pu lui infliger. Les arbres qui ont été entaillés, la libellule qui a perdu sa compagne, épinglée par l'enfant sur un mur, la chauve-souris, qui se retrouve en mari et père seul avec ses petits, la mère ayant été tuée par l'enfant. Enfin l'enfant doit faire face à l'écureuil qui a, lui, réussi à échapper à la mort et à se libérer de sa cage. L'enfant essaie alors de justifier son acte et ses paroles laissent presque entrevoir son regret et ses excuses. Autour de lui, des animaux se retrouvent en couple, se cajolent et jouent ensemble. L'enfant est alors pris de tristesse, il n'est plus le témoin effrayé de duos inquiétants qui l'accablent, il est un petit être abandonné et il appelle "maman». Mais son appel résonne encore comme une menace pour les animaux qui s'affolent et crient: "C'est l'enfant au couteau! Unissons-nous, unissonsnous!». Dans la bagarre générale, l'enfant est bousculé et l'écureuil est blessé. Il vient choir à côté de l'enfant et tout s'arrête. L'enfant s'approche alors de l'écureuil et lui panse la patte blessée. C'est la fin de son calvaire. Cette fois ce sont les bêtes qui se préoccupent de l'enfant et ce sont elles qui appellent «maman».

Le premier tableau s'ouvre sur un enfant assis à table, dans un environnement démesurément grand par rapport à lui. La précision et la richesse du texte de Colette ne laisse d'ailleurs rien au hasard:
L'EnFant:
J'ai pas envie de faire ma page,
J'ai envie d'aller me promener,
J'ai envie de manger tous les gâteaux.
J'ai envie de tirer la queue du chat
Et de couper celle de l'écureuil
J'ai envie de gronder tout le monde!
J'ai envie de mettre maman en pénitence ${ }^{10}$.

Io. Colette, Euvres, vol. 3, p. 151-169. 
La porte s'ouvre. Entre Maman (ou plutôt ce qu'en laissent voir le plafond très bas et l'échelle de tout le décor, où tous les objets assument des dimensions exagérées pour rendre frappante la petitesse de l'Enfant), c'est-à-dire une jupe, le bas d'un tablier de soie, la chaîne d'acier où pend une paire de ciseaux et une main. Cette main se lève, interroge de l'index:

Maman:

Bébé a été sage? Il a fini sa page?

Oh Tu n'as rien fait! Tu as éclaboussé d'encre le tapis! Regrettes-tu ta paresse?

D'emblée, le décalage entre la taille de l'enfant et son environnement ainsi que sa dénomination ("bébé») donnent un sens à ce contexte: l'enfant est dans une situation inconfortable. Il est tout petit et ainsi ramené à une position d'impuissance face à son environnement. Pour Mélanie Klein d'ailleurs, le gigantisme de l'environnement signait la présence de l'angoisse ${ }^{11}$. Mais qu'est-ce que l'angoisse au fond et comment émerget-elle dans cette situation? Visuellement et métaphoriquement, l'enfant n'est pas à la hauteur. Il se sent tout petit parce que la demande qui lui est faite est trop importante, trop exigeante. C'est le premier désaccord; entre son besoin du moment et les attentes de sa mère qui désire une production de grand enfant. L'enfant, face à son sentiment d'incapacité, est ainsi ramené à une position de régression, en l'occurrence celle d'un bébé. Comme le texte de Colette le souligne, l'enfant "a envie», mais cette envie peut être comprise au-delà des jugements ordinaires, qui n'y verraient qu'une une envie de faire des bêtises ou un égoïsme d'enfant capricieux. En réalité, l'envie de cet enfant est un processus plus subtil notamment décrit par Mélanie Klein. L'auteure rappelle en effet que lorsque le bébé découvre qu'il doit compter sur sa mère pour se nourrir et donc pour survivre, il fait l'expérience de sentiments puissants et violents: il voudrait avoir tout le lait, pour ne pas risquer d'en manquer, mais aussi du coup avoir la mère totalement à lui pour ne plus en être dépendant, c'est-à-dire contrôler totalement sa mère ${ }^{12}$. Comme le note Simonelli:

II. M. Klein, Essais de psychanalyse, p. 258.

I2. Id., La psychanalyse des enfants. 
L'enfant ressent de l'envie à l'égard de sa mère qui possède tout ce qu'il peut souhaiter, mais qui lui reste désespérément inaccessible. L'envie est donc accompagnée d'un sentiment de dépendance et d'insuffisance ${ }^{13}$.

C'est bien cela dont il est question dans ce premier mouvement de L'enfant et les sortilèges. L'enfant voudrait posséder sa mère, lui prendre tout (manger tous les gâteaux, gronder tout le monde, mettre maman en pénitence) et la maitriser pour ne pas devoir souffrir de ce décalage entre lui et elle. Il y a donc une tension très forte qui monte à l'intérieur de lui et qu'il ne comprend pas sur le moment, c'est bien la définition de l'angoisse. C'est-à-dire une peur qui n'est pas représentable. Cette tension peut aussi se traduire comme un tiraillement entre ce que Freud a nommé «le principe de plaisir» et «le principe de réalité»: le principe de plaisir, c'est en effet la recherche d'apaisement des tensions qui surgissent à l'intérieur de l'individu, afin de retrouver un bien-être, un équilibre psychique interne ${ }^{14}$. Le principe de réalité concerne quant à lui les adaptations de la personne aux exigences de l'environnement. Quand l'enfant est un bébé, le principe de réalité n'existe pour ainsi dire pas, car tout est centré autour des sensations internes du bébé, de son monde psychique extrêmement fragile. Plus il grandit, plus il va pouvoir organiser, mettre en forme ce monde interne pour se rendre disponible, s'éveiller de plus en plus à la réalité. Mais la condition pour qu'il puisse s'adapter à la réalité, c'est que son monde interne soit un peu régulé et organisé.

Or ici, le psychisme de cet enfant n'est pas tranquille, d'autant plus qu'il subit d'importantes pressions de la réalité, encore accentuée par la punition maternelle qui le prive de bonne nourriture ("du thé sans sucre, du pain sec») et de sa présence ("Restez tout seul jusqu'au dîner»), deux éléments justement primordiaux dans le développement du bébé. $\mathrm{Du}$ coup s'opère un retournement de situation par rapport à l'environnement imposant de cette première scène: l'enfant est débordé par ses tensions internes, il décharge sa colère en attaquant son environnement et son psychisme prend toute la place au détriment de la réalité, ce sont les sortilèges.

I3. T. Simonelli, Narcissisme destructeur et identification projective, p. 1.

I4. R. Roussillon et al., "Manuel de psychologie et de psychopathologie clinique générale», p. 30 sq. 
L'attaque de l'enfant sur les objets de son environnement s'adresse initialement à sa mère, parce qu'il est trop frustré et voudrait en quelque sorte faire disparaître celle qui est à la source de ses souffrances. L'enfant débordé par des sentiments qui sont devenus incontrôlables, passe à l'acte parce qu'il n'a plus les moyens d'exprimer sa colère autrement et de la contenir en lui. Mais celle-ci se déplace sur l'environnement et les objets qui représentent le corps de la mère. D'une part, la mère a disparu et logiquement l'enfant ne peut plus se fâcher contre elle, mais d'autre part, il est très fréquent de voir s'opérer un déplacement de l'agressivité vis-à-vis du parent sur d'autres objets: des choses ou des enfants d'ailleurs, comme la fratrie, parce que l'enfant sait au fond de lui qu'il ne fait pas le poids vis-à-vis de l'adulte et qu'il risque de détruire celui qu'il aime et dont il a besoin. Mais cela, c'est justement le problème qui va se poser pour cet enfant et sur lequel va porter la suite du récit.

La privation de la présence maternelle ou privation de holding (qualifiée ainsi par Winnicot pour décrire l'importance vitale pour le bébé d'être porté, enveloppé par le regard, la voix et les bras maternels ${ }^{15}$ ) se traduit probablement par l'apparition du fauteuil et de la bergère comme premiers objets ensorcelés, symboles d'un holding maternel défaillant, qui se dérobent justement devant l'enfant au moment même où il voudrait s'y laisser tomber:

\section{Le FAUTEUIL:}

Plus de coussins pour son sommeil,

Plus de sièges pour sa rêverie,

Plus de repos pour lui que sur la terre nue,

Et encore... qui sait?

Qui sait en effet ce qui va se jouer pour cet enfant abandonné à sa solitude et aux prises avec son monde interne totalement déstabilisé? Les cinq minutes que dure cette scène suffisent à poser les circonstances d'une dégringolade psychique survenue dans un contexte bien ordinaire. Il va falloir cependant beaucoup plus de temps et d'énergie à l'enfant pour ré-émerger de ce marasme synonyme de régression, autrement dit d'un retour en arrière, vers un stade de fonctionnement psychique archaïque.

I5. D. W. Winnicot, «Le concept d'individu sain». 
3. 1. Pourquoi les choses bougent?

L'enfant a donc attaqué un certain nombre d'objets, il s'arrête pour reprendre son souffle, et soudain les objets qu'il a martyrisés s'animent. Les sortilèges peuvent être en fait compris comme une production de l'enfant, qui perçoit le monde très différemment de ce qu'il est en réalité. Il le transforme, inconsciemment, en fonction de ce qu'il vient de vivre avec sa mère. Cette transformation du réel peut certainement nous faire penser que l'enfant rêve, ce qui serait une hypothèse probablement plus rassurante pour notre logique d'adulte. Certaines versions de l'oeuvre interprètent d'ailleurs l'apparition des sortilèges de cette manière. Mais ce n'est pourtant pas ce que Colette a écrit dans son texte initial. Elle ne précise pas que l'enfant s'endort mais que "le monde change subitement autour de lui", ce qui est une version plus intéressante et plus subtile que la proposition d'un rêve aux limites d'un réel bien circonscrit. De manière générale, Didier Houzel nous rappelle d'ailleurs que la frontière entre le dedans et le dehors d'un individu n'est pas si nette, car la perception n'est pas une photographie des objets mais « une relation au monde, qui se colore de sentiments, dont la source se trouve dans la relation à quelqu'un ${ }^{16}$.

En fait, tout le processus de construction du psychisme est un jeu d'aller et retour entre le dehors et le dedans, pour intérioriser les expériences vécues: les expériences laissent une trace (mnésique, émotionnelle) à l'intérieur et sont rejouées à l'extérieur pour être représentées, symbolisées. Le moyen par excellence qu'utilise l'enfant pour faire ce travail est le jeu c'est-à-dire le monde concret et l'agir ${ }^{17}$. Il est l'équivalent du rêve chez l'adulte. Mais dans tous les cas et à tous les âges, le rapport à la réalité n'est jamais objectif et peut s'altérer d'autant plus que le fonctionnement psychique se fragilise. Le cas le plus illustratif est sans doute le délire psychotique, dans lequel la personne hallucine des perceptions qui n'existent pas. Mais entre cette situation extrême, génératrice d'immenses angoisses et la situation idéale ou le monde fantasmatique resterait contenu dans le rêve et les pensées, l'individu, et qui plus est l'enfant qui construit son psychisme, traverse une multitude d'états et

16. D. Houzel, La transmission psychique, p. 59.

17. A. Ciccone, Y. Gauthier, B. Golse, B. Stern, Naissance et développement de la vie psychique, p. 11-37 
d'expériences dans lesquelles réalité et fantasmes sont réciproquement transformés. Ainsi, plus le fonctionnement est archaïque, plus la perception de la réalité est soumise au monde fantasmatique. Car dans l'archaïque, c'est-à-dire au début de la vie, il n'y a pas de différence pour le bébé entre intérieur et extérieur. Pas de frontières. Ce qui vient de l'autre vient de soi. De plus, le sentiment d'être soi est très fragile; le bébé éprouve des petits moments de conscience isolés, des sensations fortes qui le calment ou le désorganisent complètement. Il va donc apprendre à faire la différence entre ce qui vient de lui et ce qui vient du dehors et il va construire des représentations de ce qui se passe à l'intérieur de lui. Il lui faut donc également organiser son monde intérieur. Mais avant de pouvoir distinguer l'intérieur de l'extérieur, le bébé différencie d'abord les bons objets ou bonnes expériences des mauvais objets ou évènements qui génèrent en lui des tensions. Durant cette période, il essaie d'évacuer en dehors ce qui est désagréable et de garder ce qui est bon.

Le bébé fonctionne ainsi par mécanisme psychique de clivage, en projetant à l'extérieur et sur sa mère en particulier, les sensations indigestes et de ce fait trop angoissantes pour être assimilées. La mère n'est par conséquent pas perçue comme une personne à part entière, mais parfois comme une bonne mère, celle qui le satisfait et parfois comme une mauvaise mère, celle qui le frustre. De même, l'enfant soumis aux sortilèges est en fait débordé par ses émotions internes et ramené à un fonctionnement clivé propre au tout petit: il ne voit plus la réalité telle qu'elle est, mais telle qu'il la ressent à l'intérieur de lui-même et il sépare l'expérience vécue en bons et mauvais objets. Ce qui est violent dedans est projeté dehors sur des objets qui symbolisent le corps maternel et qui deviennent soudain menaçants pour l'enfant. Il est persécuté, mais momentanément déconnecté de ses propres actes agressifs. Ce phénomène de clivage avait frappé Mélanie Klein lorsqu'elle s'intéressa à l'opéra en 1929 mais ce n'est que plusieurs années après qu'elle nommera ce processus psychique «identification projective», soit la possibilité de mettre au dehors de soi des éléments psychiques indigestes, violents et inquiétants, pour tenter de s'en protéger ${ }^{18}$.

A l'époque, Mélanie Klein n'a jamais approfondi le rôle de la mère face à ces attaques du bébé, mais d'autres psychanalystes ont par la suite cherché à comprendre l'importance de ce mécanisme dans le

I8. C. Bolgert, «L'identification projective». 
développement. C'est principalement W. Bion qui émit l'hypothèse que cette projection du bébé sur sa mère était en fait une sort d'appel adressé à un autre sujet, capable de transformer ces pulsions violentes, parce que non représentées, en pensées supportables ${ }^{19}$.

Dans son livre sur la transmission psychique, Didier Houzel interroge ainsi la question de l'absence de la mère dans l'œuvre de Colette et Ravel: que se serait-il passé si la mère de cet enfant était restée pour accueillir ses tensions? L'angoisse aurait-elle peut-être pu redescendre sans que l'enfant doive se mettre "hors de lui ${ }^{20}$ ?

Ici pourtant l'enfant est bel et bien seul face à ses productions inquiétantes et c'est en lui et avec peine qu'il va devoir trouver les moyens de se réorganiser.

Parmi les différentes scènes de rétorsion des objets à l'égard de l'enfant, la vengeance de l'arithmétique est d'ailleurs un bon exemple de l'effet de "déliaison» du mécanisme de clivage: l'enfant n'arrive plus à faire de liens dans ses apprentissages et à y trouver un peu de plaisir. Ce qu'il a appris devient incompréhensible, car à trop vouloir séparer le bon du mauvais, tous les liens sont attaqués et les pensées perdent leur sens:

L'ENFANT (affolé):

Mon Dieu! C'est l'Arithmétique!

Le PETIT VIEILLARD, LES CHIFFres: (soulevant les feuillets et piaillant)

Tique, tique, tique!

(Il danse autour de l'Enfant en multipliant les passes maléfiques.)

Le Petit Vieillard (en se pinçant le nez): Quatre et quat'dix-huit,

Onze et six vingt-cinq, Quatre et quat'dix-huit,

Sept fois neuf trente-trois

L'ENFANT (surpris): Sept fois neuf trente-trois?

Les chiffres:

Sept fois neuf trente-trois, etc. (ils sortent de dessous les feuillets)

L'ENFAnt (égaré): Quatre et quat'

LE PETIT VIEILLARD (soufflant):

Dix-huit!

LENFANT:

Onze et six?

Le Petit Vieillard (même jeu): Vingt-cinq!

L'Enfant (exagérant résolument): Trois fois neuf quat'cent!

I9. W. Bion, Aux sources de l'expérience.

20. D. Houzel, La transmission psychique, p. 64. 
Le PeTit VieILlard (Il se balance pour prendre le mouvement de la ronde): Millimètre, Centimètre, Décimètre,

Décamètre, Hectomètre, Kilomètre, Myriamètre, Faut t'y mettre!

Quelle fêtre! Des millions, Des billions, Des trillions, et des frac-cillions!

\section{2. Mais qu'est-ce qui angoisse aussi l'enfant?}

Pour résumer très brièvement cette question du développement du bébé, on peut donc dire que le duo mère-enfant commence par une symbiose et évolue vers une différenciation du bébé vis-à-vis de sa mère.

Ce sont les décalages ou désaccords dans cette union (la mère qui n'est pas là quand le bébé la réclame ou qui répond à côté des besoins de l'enfant) qui à la fois font prendre conscience au bébé qu'il n'est pas un mais qu'ils sont deux et génèrent du même coup des angoisses, qui sont des réactions aux désirs d'attaquer cette maman insatisfaisante.

On voit ainsi que dans cette première partie de l'opéra, comme dans la vie du bébé, la haine et l'amour éprouvées pour la mère ne sont pas supportables ensemble. L'enfant se fait en quelque sorte une représentation double de sa mère: la mauvaise mère ou la bonne mère mais pas les deux.

En plus de la découverte de cette relation à deux, le petit enfant va rapidement se poser des questions sur ses origines et sur le couple parental. Il se construit alors des fantasmes autour de la sexualité et essaie d'imaginer ce qui se passe entre ses parents. Mais l'image qu'il se fait de cette union, couramment nommée "la scène primitive", est une représentation souvent inquiétante, parce que l'enfant ne comprend pas vraiment ce qu'il s'y passe et parce qu'il se la représente à partir de ses propres fantasmes de tout petit, de ses propres expériences de désir si intense vis-à-vis de sa mère. Il s'imagine donc souvent quelque chose de violent, où il y a une agression du père envers la mère, où l'un élimine l'autre.

Ici quasiment tous les objets qui s'animent évoquent ces duos inquiétants: le fauteuil et la bergère, la tasse et la théière, le feu et la cendre, le chat et la chatte et d'autres encore.

Une scène en particulier, celle du feu et de la cendre exprime avec grande finesse ce jeu amoureux entre deux êtres qui se solde par l'anéantissement de l'un des deux: 
Derrière le Feu, née sous ses pas, monte la Cendre. Elle est grise, onduleuse, muette, et le Feu ne la voit d'abord pas. Puis, l'ayant vue il joue avec elle. Elle joue avec lui. Elle tente, sous ses longs voiles gris, de maîtriser le Feu. Il rit, s'échappe et danse. Le jeu continue jusqu'au moment où, las de lutter, le Feu se laisse éteindre. Il tente un dernier sursaut pour se libérer, brille encore un instant, puis s'endort, roulé dans les longs bras et les longs voiles.

Cette confrontation à la scène primitive a un pouvoir désorganisateur en même temps qu'elle comporte en elle la clé d'une réorganisation psychique. Dans la scène du jardin, va d'ailleurs se mettre en forme une autre dimension du couple, celle du lien où la question de l'attachement devient plus forte que la question de l'intrusion. C'est le couple qui s'aime et dont l'enfant est exclu, l'enfant n'est plus un voyeur sidéré par une scène qu'il ne comprend pas, mais un être seul face au couple c'està-dire limité (fille ou garçon mais pas les deux, enfant de ses parents et pas l'inverse).

\section{3. Comment l'enfant s'en sort-il?}

L'enfant emporté dans son monde fantasmatique se trouve pris dans la répétition de plusieurs scènes similaires avant de pouvoir transformer les représentations qui l'assaillent. L'histoire qui avait débuté «l'après-midi » dans la description de Colette, se poursuit dans la nuit jusqu'à l'aube réconfortante qui annoncera les retrouvailles avec sa mère. Le temps s'est suspendu avec la destruction du balancier de l'horloge et a laissé place au règne de l'inconscient qui "ignore le temps" selon les termes de Freud. La nuit qui s’installe évoque aussi la perte des repères de la réalité et la frayeur de l'enfant. Celui-ci, d'abord impuissant et sidéré par les objets qui le menacent, va chercher cependant quelque chose de bon en lui qui puisse le soutenir et va pouvoir redevenir actif dans son jeu imaginaire.

C'est d'abord le lien bénéfique à la princesse des contes de fées qu'il tente de faire exister, mais qui ne tient malheureusement pas. Il a ensuite un instant de joie lorsqu'il sort dans le jardin, mais les arbres se plaignent de leurs blessures et tout redevient inquiétant et sombre. Pourtant l'enfant réagit cette fois et ose questionner: "Quelles blessures?», un premier mouvement pour faire face à ses actes de destructions. Il répond ensuite à la libellule qui cherche sa compagne «La libellule que j’ai prise... Percée 
d'une épingle... contre le mur. Ah!...", explication qui signe sa capacité à reconnaitre ces actes agressifs comme les siens.

Mais plus que d'assumer ses actes agressifs, il en retrouve un sens plus humain (le désir d'explorer et de comprendre plutôt qu'une malveillance gratuite), ce qui le rend moins mauvais lorsqu'il essaie de justifier son geste à l'écureuil qu'il avait capturé: «La cage c'était pour mieux voir ta prestesse, tes quatre petites mains, tes beaux yeux...».

Dans ce passage, on voit que pour l'enfant, l'autre, en l'occurrence l'écureuil, devient un peu comme lui : il peut le voir tel qu'il est, reconnaître à l'écureuil des bons côtés et reconnaître en lui ses actes agressifs. C'est une diminution de la force projective.

Il est d'ailleurs probable qu'il peut le faire avec l'écureuil parce qu'il n'a pas été trop loin dans l'agression de ce dernier. Il peut s'accrocher à quelque chose de suffisamment positif dans ce lien pour pouvoir avoir de l'empathie avec cet animal et pour pouvoir, de manière plus générale, se réconcilier avec le jardin. Selon Gianna Williams, il est en effet très difficile d'atteindre un sentiment de compassion «quand on sent que le dommage auquel on est confronté est énorme et catastrophique» ${ }^{21}$.

Il y a ainsi pour cet enfant juste assez d'objets bienveillants (la princesse, le jardin, les animaux qui deviennent moins sévères et qui retrouvent leur vitalité) pour permettre une transformation de son fonctionnement psychique. D'ailleurs, après l'échange avec l'écureuil, les animaux se mettent à jouer, à s'aimer, à s'animer joyeusement et l'aube paraît en arrière-plan :

Pendant qu'il parle, le jardin se peuple d'écureuils bondissants. Leurs jeux, leurs caresses, suspendues en l'air, n'inquiètent pas ceux des rainettes, au-dessous. Un couple de sphinx de laurier-rose les imite. D’autres groupes se nouent, se défont. Le jardin, palpitant d'ailes, rutilant d'écureuils, est un paradis de tendresse et de joies animales.

Curieusement, cette joie des couples et du groupe heureux déprime l'enfant. On touche là à un point majeur dans son évolution, le fait de reconnaître ses limites (incomplétude) et sa solitude. Solitude face au couple parental qui a, forcément, vécu quelque chose avant lui (la scène primitive) et qui demeure à jamais inaccessible. La reconnaissance et l'acceptation d'un couple lié face à l'enfant, c'est à la fois sur un plan

2I. G. Williams, «Pensées sur la position dépressive». 
fantasmatique, l'acceptation que le bon et le mauvais puissent aller ensemble et, sur le plan de la réalité, l'acceptation de ses propres limites: l'enfant comprend alors que la personne qu'il aime et qu'il déteste est une seule personne et que s'il élimine le mauvais objet il élimine aussi le bon objet. On ne peut pas tout faire, sans risquer de détruire celui/ ceux qu'on aime et sans risquer de se détruire soi-même. C'est bien de cela qu'il s'agit dans le dernier passage de L'enfant et les sortilèges. Lorsque l'enfant appelle maman, les animaux fondent sur lui dans une bagarre générale et l'écureuil est blessé en même temps que l'enfant. On voit là comment l'enfant a pu s'identifier à l'écureuil (il me ressemble, je suis comme lui, ce qui lui arrive pourrait m'arriver à moi) et c'est probablement pour cela que les deux sont blessés. L'enfant est cette fois authentiquement touché par ce qu'il a pu faire à sa mère et veut tout faire pour réparer ses méfaits.

L'enfant soigne alors l'écureuil sous le regard ému et abasourdi des animaux qui chantent «il a lié la patte» une expression qui peut surprendre, là où l'on s'attendrait à lire «il a pansé» ou «soigné la patte». Mais ce choix de Colette du verbe «lier» résonne profondément avec la dimension du lien mère-enfant évoquée précédemment et avec le fonctionnement psychique de l'enfant jusque-là clivé (autrement-dit "dé-lié»). En liant la patte de l'écureuil, l'enfant répare l'agression qu'il a commise et peut reconnaitre sa mère telle qu'elle est réellement, à la fois gratifiante et frustrante. C'est alors au tour des animaux d'appeler «maman» pour que l'enfant puisse la retrouver.

Colette termine son livret par cette description magnifique de l'enfant prêt à rejoindre sa mère:

Une lumière parait aux vitres, dans la maison. En même temps, la lune dévoilée, l'aube, rose et d'or, inondent le jardin d'une clarté pure. [...] Les bêtes l'escortent [l'enfant] d'un peu plus loin, le fêtent de battements d'ailes, de culbutes de joie, puis, limitant à l'ombre des arbres leur bienveillant cortège, laissent l'enfant seul, droit, lumineux et blond, dans un halo de lune et d'aube, et tendant les bras vers celle que les Bêtes ont appelée: «Maman».

Au final, le mouvement psychique qui a lieu dans cette histoire est le passage d'une position de toute-puissance infantile à une intériorisation de liens et de limites. 
Cette étape a été nommée par Mélanie Klein, "position dépressive " ${ }^{22}$. Même s'il ne s'agit pas dans ce cas d'une dépression de l'enfant telle qu'on la conçoit habituellement, c'est-à-dire comme une maladie, la position dépressive décrit un passage normal et sain du développement de l'enfant, mais un passage que ne se fait pas sans souffrance.

L'enfant est dans ces moments confronté à la finitude de toute chose, au risque de perte, à son potentiel destructeur et à ses limites. Mais c'est aussi à partir de là qu'il va pouvoir développer son potentiel créateur. La réparation implique la recréation de l'objet, puisque celui-ci n'est plus le même qu'avant et produit un apaisement de la culpabilité. Pour certains psychanalystes, ce serait donc dans ce processus de réparation que naitrait l'acte créatif, et de cette manière, la possibilité de transformer les pulsions agressives ${ }^{23}$.

Pour conclure sur cette œuvre magnifique, on ne peut que revenir à Colette et à Ravel eux-mêmes, à cette création qui fut la leur, dans un moment de vie marqué pour chacun d'eux par la disparition de leur mère.

Et si l'enfant et ses sortilèges était à la fois un peu Colette, à la fois un peu Ravel, deux grands enfants privés de leur mère, décédée trop tôt? Deux enfants avec leur chagrin, leur colère d'avoir perdu cet être tant aimé, en panne devant leur travail de création. Et si c'était Colette et Ravel qui avaient dû affronter seuls les fantasmes de leurs esprits endeuillés? Peut-on imaginer que ces deux artistes aient dû aussi chercher à l'intérieur d'eux quelque chose de bon qui puissent les aider à dépasser ce sentiment de perte de l'être aimé et à transformer leur deuil en création?

Laurence LACOUR

Institution de Lavigny

22. M. Klein, Essais de psychanalyse 1921-1945, p. 341-369.

23. H. Segal, Délire et créativité. 


\section{BIBLIOGRAPHIE}

Bion, Wilfred R., Aux sources de l'expérience, Paris, PUF, 1979.

Bolgert, Catherine, "L'identification projective», Gestalt, 24 (2003), p. 141-159.

Brussel, René, "Ravel, classique français", in Maurice Ravel Qui êtesvous?, Lyon, La Manufacture, 1987, p. 72-76.

Cicconne, Albert, Gauthier, Yvon, Golse, Bernard, Stern, Daniel N., Naissance et développement de la vie psychique, Toulouse, Erès, 2008.

Colette, Euvres, Paris, Gallimard, 1991, vol. 3 (Bibliothèque de la Pléiade).

Colette, Delage, Maurice, Fargue, Léon-Paul, JourdanMorhange, Hélène, Klingsor, Tristan, Roland-Manuel, Sordet, Jean-François André, Vuillermoz, Emile, De Zogheb, Jacques, Maurice Ravel par quelques-uns de ses familiers, Paris, Editions du Tambourinaire, 1939.

Delcroix, Maurice, "De la maison de Sido à la Maison de Claudine", in Colette, dir. par Gérard Bonal et Frédéric Maget, Paris, Ed. de l'Herne, 2011, p. 13-18 (Cahiers de l'Herne, 97).

Houzel, Didier, La transmission psychique, Paris, Odile Jacob, 2010. KLEIn, Mélanie, Essais de psychanalyse 1921-1945, Paris, Payot, 1968.

—, La psychanalyse des enfants, Paris, PUF, 2013 (Quadrige).

Pichois, Claude et Vincenette, Album Colette, Paris, Gallimard, 1984.

Pourvoyeur, Robert, "Sortilèges de Colette et Ravel», L'Avant-Scène Opéra, 127 (1990), p. 18-22.

Roussillon, René, Chabert, Catherine, Ciccone, Albert, Ferrant, Alain, Georgieff, Nicolas, Roman, Pascal, Manuel de psychologie et de psychopathologie clinique générale, Issy-les-Moulineaux, Elsevier Masson, 2007.

Sanson, David, Maurice Ravel, Arles, Actes Sud, 2005.

SEgal, Hanna, Délire et créativité - Essais de psychanalyse clinique et théorique, Paris, Des Femmes, 1987. 
Simonelli, Thierry, Narcissisme destructeur et identification projective (2015) (http://www.psychanalyse.lu/articles/ SimonelliNarcissismeProjection.htm).

Vuillermoz, Emile, "Maurice Ravel» in Maurice Ravel Qui êtes-vous? Lyon, La Manufacture, 1987, p. 109-117.

Williams, Gianna, «Pensées sur la position dépressive» in Dépression $d u$ bébé, dépression de l'adolescent, éd. par Alain Braconnier, Bernard Golse, Toulouse, ERES, 2010, p. 139-155.

Winnicot, Donald Woods, "Le concept d'individu sain", in Conversations ordinaires, Paris, NRF Gallimard, 1988, p. 23-42 (Connaissance de l'Inconscient). 
